\title{
The mNCP-SPI Score Predicting Risk of Severe COVID-19 among Mild-Pneumonia Patients on Admission
}

This article was published in the following Dove Press journal: Infection and Drug Resistance

\begin{abstract}
Lingxi Guo, (iD 1,2,* Weining Xiong, ${ }^{3, *}$ Dong Liu, 1,2,* Yun Feng, (iD 1,2,* Peng Wang, ${ }^{4, *}$ Xuan Dong, ${ }^{5, *}$ Rong Chen, ${ }^{1,2}, *$ Yi Wang, ${ }^{6}$ Lei Zhang, ${ }^{6}$ Jingwen Huang, ${ }^{1,2}$ Hanssa Dwarka Summah, ${ }^{7}$ Fangying Lu, ${ }^{1,2}$ Yusang Xie, (iD ${ }^{1,2}$ Huihuang Lin, ${ }^{1,2}$ Jiayang Yan, ${ }^{1,2}$ Hongzhou Lu, (ID) ${ }^{8}$ Min Zhou, ${ }^{1,2}$ Jieming $\mathrm{Qu}^{1,2}$
\end{abstract}

'Department of Respiratory and Critical Care Medicine, Ruijin Hospital, Shanghai Jiao Tong University School of Medicine, Shanghai, People's Republic of China; ${ }^{2}$ Institute of Respiratory Diseases, Shanghai Jiao Tong University School of Medicine, Shanghai, People's Republic of China; ${ }^{3}$ Department of Respiratory and Critical Care Medicine, Ninth People's Hospital, Shanghai Jiao Tong University School of Medicine, Shanghai, People's Republic of China; ${ }^{4}$ Departement of Respiratory and Critical Care Medicine, Baoshan Branch of Shanghai First People's Hospital, Shanghai, People's Republic of China; ${ }^{5}$ Tuberculosis and Respiratory Department, Wuhan Jinyintan Hospital, Wuhan, People's Republic of China; ${ }^{6}$ Department of Respiratory and Critical Care Medicine, Key Laboratory of Pulmonary Diseases of Health Ministry, Key Cite of National Clinical Research Center for Respiratory Disease, Wuhan Clinical Medical Research Center for Chronic Airway Diseases, Tongji Hospital, Tongji Medical College, Huazhong University of Sciences and Technology, Wuhan, People's Republic of China; ${ }^{7}$ Chest Department, Poudre D'Or Chest Hospital, Riviere du Rempart, Mauritius; ${ }^{8}$ Department of Infectious Disease, Shanghai Public Health Clinical Center, Shanghai, People's Republic of China

*These authors contributed equally to this work

Correspondence: Jieming Qu Department of Respiratory and Critical Care Medicine, Ruijin Hospital, Shanghai Jiao Tong University School of Medicine, Shanghai 20025, People's Republic of China Email jmqu0906@163.com

Min Zhou

Department of Respiratory and Critical Care Medicine, Ruijin Hospital, Shanghai Jiao Tong University School of Medicine, Shanghai 20025, People's Republic of China Email doctor zhou99@163.com
Purpose: To predict the risk of developing severe pneumonia among mild novel coronavirus pneumonia (mNCP) patients on admission.

Methods: A retrospective cohort study was conducted at three hospitals in Shanghai and Wuhan from January 2020 to February 2020. Real-time polymerasechain-reaction assays were used to detect COVID-19. A total of 529 patients diagnosed with NCP were recruited from three hospitals and classified by four severity types during hospitalization following the standards of the Chinese Diagnosis and Treatment of Pneumonia Caused by New Coronavirus Infection (eighth version). Patients were excluded if admitted by ICU on admission ( $n=92$, on a general ward while meeting the condition of severe or critical type on admission $(n=25)$, or there was insufficient clinical information $(n=64)$. In sum, 348 patients with mNCP were finally included, and 68 developed severe pneumonia.

Results: $\mathrm{mNCP}$ severity prognostic index values were calculated based on multivariate logistic regression: history of diabetes (OR 2.064, 95\% CI 1.010-4.683; $p=0.043$ ), time from symptom onset to admission $\geq 7$ days (OR 1.945, 95\% CI 1.054-3.587; $p=0.033$ ), lymphocyte count $\leq 0.8$ (OR 1.816, 95\% CI 1.008-3.274; $p=0.047$ ), myoglobin $\geq 90 \mathrm{mg} / \mathrm{L}$ (OR 2.496, 95\% CI 1.235-5.047; $p=0.011$ ), and D-dimer $\geq 0.5 \mathrm{mg} / \mathrm{L}$ (OR 2.740, 95\% CI $1.395-5.380 ; p=0.003)$. This model showed a $c$-statistics of 0.747 , with sensitivity and specificity 0.764 and 0.644 , respectively, under cutoff of 165 .

Conclusion: We designed a clinical predictive tool for risk of severe pneumonia among mNCP patients to provided guidance for medicines. Further studies are required for external validation.

Keywords: novel coronavirus pneumonia, predicting score, severe pneumonia

\section{Introduction}

On December 8, 2019, a cluster of cases of pneumonia emerged in Wuhan, Hubei province, China. ${ }^{1,2}$ High-throughput sequencing of isolates obtained from patients with pneumonia identified a novel coronavirus (COVID-19) as the etiology, 3,4 which resembled severe acute respiratory syndrome coronavirus (SARS-CoV). $3,5,6$ As of February 22, 2020, a total of 76,395 patients had been confirmed to be affected with COVID-19 in China. Significantly, it is estimated that about $17 \%$ of patients have developed severe pneumonia, 5\%-32\% admitted to intensive care units (ICUs), and 1.36\%-15\% succumbed, according to different reports. ${ }^{1,7} \mathrm{~A}$ variety of studies have shown that some clinical indicators have prognostic value in novel $\mathrm{CoV}$ pneumonia (NCP), ${ }^{1,8,9}$ and several predictive scores, such as the 
pneumonia severity index, ${ }^{10}$ CURB-65, ${ }^{11}$ and some ICU scoring systems, including Acute Physiology and Chronic Health Evaluation II score ${ }^{12}$ and Sequential Organ-Failure Assessment, ${ }^{13}$ were existed to assess the severity of pneumonia. However, none of these indices has been reported to predict the prospective severe exacerbations of NCP. Due to the seriousness of the epidemic, it is emergent to search for an early-warning model for severe NCP to assist clinicians in deciding on optimal therapeutic interventions. Herein, we unravel potential risk factors and develop a simple and practical score to predict diseaseprogression probability of mild NCP (mNCP) patients that can accurately stratify hospitalized patients with COVID-19 into relevant risk categories and provide guidance to make further clinical decisions.

\section{Methods}

\section{Study Design and Population}

This was a retrospective multicenter observational cohort study conducted from January 2020 to February 2020 in Ruijin Hospital (affiliated with Shanghai Jiao Tong University School of Medicine), Shanghai Public Health Clinical Center in Shanghai, and Jinyintan Hospital in Wuhan, China. The latter two are special designated local hospitals for NCP patients. The study was approved by the Shanghai Public Health Clinical Center Ethics Committee, Ruijin Hospital Ethics Committee, and Jinyintan Hospital Ethics Committee, respectively, while consent was obtained from the participants in our study.

All patients who had been diagnosed with NCP were consecutively enrolled from the three hospitals following WHO guidelines. Patients were divided into four types during overall hospitalization - mild, common, severe, and critical - according to the standards set by the Chinese Diagnosis and Treatment of Pneumonia Caused by New Coronavirus Infection (Eighth Version) as follows (assessors of the classification were blind to the final prediction scores in this study):

1. Mild: patients with mild clinical symptoms and no radiological abnormality

2. Common: patients presented with fever, cough or other respiratory symptoms, and positive radiological findings for pneumonia

3. Severe: if one of the following conditions were met: 1. Respiratory distress $\geq 30$ per minute
2. Finger oxygen saturation $\left(\mathrm{SaO}_{2}\right) \leq 93 \%$ in resting state

3. Partial pressure of arterial oxygen $\left(\mathrm{PaO}_{2}\right) /$ concentration of oxygen inhaled $\left(\mathrm{FiO}_{2}\right) \leq 300 \mathrm{mmHg}$

4. Clinical symptoms had worsened progressively and pulmonary lesion had progressed significantly — > $>50 \%$ on thoracic imaging within 24-48 hours

4. Critical: if one of the following conditions was met:

1. Respiratory failure and mechanical ventilation needed

2. Shock

3. Patients with other organ dysfunction needing ICU monitoring and treatment

Patients meeting the clinical characteristics of either the mild or common type were identified as $\mathrm{MNCP}$ and thus enrolled in this study. Patients were excluded if admitted to an ICU, on a general ward while meeting the conditions of severe or critical types on admission, and there was a lack of clinical information $\geq 60 \%$.

\section{Data Collection}

We collected epidemiological, demographic, clinical, laboratory, management, and outcome data from NCP patients' medical records from the three hospitals. Clinical outcomes were followed up to February 21, 2020. All data were checked by two physicians. A confirmed case with COVID-19 was defined as a positive result to high-throughput sequencing orRTPCR assay for throa- swab specimens from the upper respiratory tract that had been obtained from all patients using established techniques. ${ }^{7}$ Clinical information and classification of severity on admission were available to the statisticians.

\section{Outcomes}

The outcome focused on in this study was patients progressing to severe NCP, defined as developing to severe or critical during hospitalization. We divided patients into two groups: severe for mNCP patients who developed severe NCP and nonsevere for other patients who remained as $\mathrm{mNCP}$ before discharge.

\section{Statistical Methods}

Normally distributed continuous variables are expressed as means $\pm \mathrm{SD}$ and were compared using $t$-tests. Continuous 
variables with skewed distribution are shown as medians (IQR) and compared using the Mann-Whitney test. Categorical variables are presented as numbers and percentages and compared using the $\chi^{2}$-test. The significance of each variable was initially assessed by univariate analysis by comparing the severe group and the nonsevere group. Collinearity diagnosis was performed by calculating the variance-inflation factor (VIF) of each predictor. Variables with VIF $\geq 2$ were excluded from multivariate analysis. Variables with $p<0.1$ were regarded as potential risk factors and included in multivariate regression analysis reduced by a backward-elimination procedure (conditional likelihood-ratio test and elimination if $p \geq 0.05$ ). Continuous variables with statistical significance were categorized and retained for multivariate testing, and cutoff points were identified following Youden's index of the receiver-operating characteristic (ROC) curve or a clinically relevant cutoff.

Prognostic indices were calculated based on the results of the multivariate analysis. The performance of the score was assessed by measuring the area under the ROC (AUROC), while sensitivity and specificity were also calculated. Internal validation was performed by applying the 2,000 bootstrap-resampling technique. The 2,000 samples were randomly assigned to two complementary subsets: the training set and the testing set, while the AUROC was measured for each subset. ROC curves and net reclassification improvement (NRI) analyses were used to assess improvement in risk prediction compared with CURB-65.

Validity was retested using fivefold cross-validation in the derivation cohort, which was divided randomly into five subsamples. A logistic regression model was developed using four subsamples and the score then calculated using the remaining (held out) subsample. This process was repeated for each of the five loops of cross-validation so that in the end each patient had a prediction score, for which overall (cross-validated) association and inference was tested. Statistical analysis was performed using SPSS version 22.0 and $\mathrm{R}$ 3.5.0. All tests were two-sided, and $p<0.05$ denoted statistical significance.

\section{Results \\ Patient Characteristics}

From January to February 2020, 529 patients diagnosed with NCP were recruited as eligible participants from the three hospitals and classified by four severity types during overall hospitalization. Among them, 92 patients had initially been admitted by an ICU, 25 were in a general ward while meeting the condition of severe or critical on admission, and 64 patients lacking clinical information $\geq 60 \%$ were excluded. We finally included 348 patients with $\mathrm{mNCP}$, while 68 cases (19.5\%) developed to severe or critical during hospitalization. Baseline characteristics of all patients and comparisons between severe and nonsevere groups are described in Supplemental Table 1. Comparison between participants with complete and incomplete data is in Supplemental Table 2.

Mean interval between onset of symptoms and admission was $7.77 \pm 5.59$ days, while patients from the severe group showed significantly longer mean waiting time of 9.68 days compared with 7.31 days in the nonsevere group $(p=0.002)$. A total of 119 patients $(34.2 \%)$ had comorbidities, with hypertension being the most commonly observed $(23.6 \%)$, followed by diabetes, coronary heart disease, and cancer. Overall, higher proportions of history of cardiocerebral vascular disease, diabetes, and cancer were observed in patients in the severe group than the nonsevere group.

Laboratory findings comprising leukocytes, neutrophils, total bilirubin, direct bilirubin, urea, myoglobin, D-dimer, fibrinogen, and CRP levels were obviously increased in the severe group over the nonsevere group. The severe group showed large variability in lymphocyte counts, with higher levels of lymphopenia (lymphocytes $\leq 0.8 \times 10^{9} / \mathrm{L}$ ). Hypoxemia was defined as $\mathrm{PaO}_{2} \leq 60 \mathrm{mmHg}$ and hyperoxia $\mathrm{PaO}_{2} \geq 120 \mathrm{mmHg}$ according to arterial blood gas, the latter appearing in $25.1 \%$ of patients in the common group. We counted lung-lobe infiltrations according to chest CT, and the mean value was 3.67 (only five lung lobes in total), indicating extensive involvement of NCP.

For coinfection, two patients were infected with EpsteinBarr virus, while no other respiratory virus infection was found. Only four hospitalized patients had bacterial coinfection. Two patients from the nonsevere group were infected with antibiotic-resistant bacteria, which were Escherichia coli and Serratia marcescens separately. One other patient was positive for Mycoplasma pneumoniae. No infection of fungi or tuberculosis was found among the patients.

As for treatment, antivirus therapy in the first 4 days after onset of symptoms was noted as early antivirus treatment, and was applied for probably half the patients in our study. Despite the low cobacterial infection rate, preventive antibiotic therapy was applied for $70 \%$ of our patients. The severe group had higher probabilities of both antibiotic and intravenous corticosteroid therapies. In our study, 23 patients died during hospital stay, indicating 
Table I Comparison of T-lymphocyte Subtypes, Humoral Immunity, and Cytokines Between Severe and Nonsevere Groups

\begin{tabular}{|c|c|c|c|c|}
\hline & Total $(n=200)$ & Nonsevere $(n=181)$ & Severe $(n=19)$ & $p$-value \\
\hline $\mathrm{CD}^{+}(\%)$ & $29.07 \pm 29.22$ & $29.32 \pm 30.31$ & $26.55 \pm|4.5|$ & 0.719 \\
\hline $\mathrm{CD}^{+}(\%)$ & $66.78 \pm 13.18$ & $67.42 \pm 13.11$ & $60.25 \pm 12.44$ & 0.038 \\
\hline $\mathrm{CD}^{+}(\%)$ & $41.91 \pm 20.09$ & $42.44 \pm 20.73$ & $36.43 \pm 10.83$ & 0.255 \\
\hline Absolute CD3 count & $301.35 \pm 180.37$ & $311.01 \pm 179.98$ & $207.63 \pm \mid 59.91$ & 0.017 \\
\hline Absolute CD4 count & $809.23 \pm 432.00$ & $839.30 \pm 429.62$ & $522.84 \pm 349.04$ & 0.002 \\
\hline Absolute CD8 count & $482.46 \pm 262.55$ & $498.69 \pm 261.91$ & $327.00 \pm 218.86$ & 0.006 \\
\hline $\mathrm{CD}^{+}: \mathrm{CD}^{+}$ratio & $1.75 \pm 0.73$ & $1.75 \pm 0.75$ & $1.75 \pm 0.44$ & 0.967 \\
\hline $\lg G$ & $12.06 \pm 2.57$ & $12.05 \pm 2.49$ & $12.14 \pm 3.43$ & 0.893 \\
\hline $\lg A$ & $2.53 \pm 0.99$ & $2.50 \pm 0.97$ & $2.86 \pm 1.16$ & 0.187 \\
\hline $\lg M$ & $1.02 \pm 0.50$ & $1.02 \pm 0.5 \mid$ & $0.95 \pm 0.48$ & 0.606 \\
\hline IL6 & $11.07 \pm 13.10$ & $10.30 \pm 8.99$ & $12.44 \pm 18.29$ & 0.367 \\
\hline
\end{tabular}

a mortality rate of $6.6 \%$ for $\mathrm{mNCP}$ patients. All deaths occurred in the severe group. Eleven patients were admitted to an ICU before death. Immunologic tests between severe and nonsevere NCP patients are described in Table 1. Lower serum levels of $\mathrm{CD}^{+}, \mathrm{CD}^{+}$, and $\mathrm{CD}^{+}$ T-lymphocyte subtypes were noted in the severe group.

\section{Risk Factors of Developing Severe Pneumonia}

According to the aforementioned methods and analyses, 13 categorical variables were entered in a backward stepwise logistic regression analysis (Table 2). The optimal feature subset selection showed that a model with five predictors had the minimum Bayesian information criterion, while the best subset features turned out to be diabetes, interval between onset of symptoms and admission, lymphocyte count, myoglobin, and D-dimer. The MSE value of the testing data set was 0.488 .
According to the logistic regression model, five parameters were included as predictive factors (Table 2): history of diabetes (OR 2.064, 95\% CI 1.010-4.683; $p=0.043$ ), time from symptom onset to admission $\geq 7$ days (OR 1.945, 95\% CI 1.054-3.587; $p=0.033$ ), lymphocyte count $\leq 0.8 \times 10^{9} / \mathrm{L}$ (OR 1.816, 95\% CI 1.008-3.274; $p=0.047)$, myoglobin $\geq 90 \mathrm{ng} / \mathrm{mL}$ (OR 2.496, 95\% CI 1.235-5.047; $p=0.011$ ), and D-dimer $\geq 0.5 \mathrm{mg} / \mathrm{L} \quad(\mathrm{OR}$ $2.740,95 \%$ CI 1.395-5.380; $p=0.003)$. The VIF of each variable was $<2.0$. The score was named the mNCP severity prognostic index (mNCP-SPI).

\section{Prognostic Index and Validation}

Relative weights were assigned according to the regression coefficient of each categorical variable $(\beta)$ Prognostic score was calculated as follows:

Predictive score $=73.40221$ (if history of diabetes)

Table 2 Univariate and Multivariate Analysis Associated with In-Hospital Severity Progression of Patients with mNCP

\begin{tabular}{|c|c|c|c|c|}
\hline \multirow{2}{*}{$\begin{array}{l}\text { Clinical feature } \\
\text { Male }\end{array}$} & \multicolumn{2}{|l|}{ Univariate } & \multicolumn{2}{|l|}{ Multivariate } \\
\hline & $1.638(0.936-2.868)$ & 0.084 & & \\
\hline Age $\geq 65$ years & $2.000(1.119-3.573)$ & 0.019 & & \\
\hline Time from symptom onset to admission $\geq 7$ days & $2.370(1.346-4.17 I)$ & 0.003 & $1.945(1.054-3.587)$ & 0.033 \\
\hline Cardiovascular diseases & $1.822(1.043-3.185)$ & 0.035 & & \\
\hline Diabetes & $2.286(1.079-4.843)$ & 0.031 & $2.064(1.010-4.683)$ & 0.043 \\
\hline Cancer & $4.365(1.227-15.535)$ & 0.023 & & \\
\hline Lymphocytes $\leq 0.8 \times 10^{9} / \mathrm{L}$ & $2.625(1.552-4.525)$ & 0.001 & $1.816(1.008-3.274)$ & 0.047 \\
\hline Albumin $\leq 30 \mathrm{~g} / \mathrm{L}$ & $3.947(2.138-7.286)$ & $<0.001$ & & \\
\hline Urea $\geq 7 \mathrm{mmol} / \mathrm{L}$ & $2.160(1.074-4.347)$ & 0.031 & & \\
\hline Myoglobin $\geq 90 \mathrm{ng} / \mathrm{mL}$ & $3.904(2.027-7.519)$ & $<0.001$ & $2.496(1.235-5.047)$ & 0.011 \\
\hline D-dimer $\geq 0.5 \mathrm{mg} / \mathrm{L}$ & $4.325(2.297-8.144)$ & $<0.001$ & $2.740(1.395-5.380)$ & 0.003 \\
\hline $\mathrm{PaO}_{2} \leq 80 \mathrm{mmHg}$ & $\mid 5.947(6.556-38.79 \mid)$ & $<0.001$ & & \\
\hline Multilobe infiltrates $\geq 4$ & $5.611(1.903-16.545)$ & 0.002 & & \\
\hline
\end{tabular}


Points

$\begin{array}{lllllllllll}0 & 10 & 20 & 30 & 40 & 50 & 60 & 70 & 80 & 90 & 100\end{array}$

Diabetes

Time from symptom onset

to admission

No

Yes

$\geq 7$ days

Lymphocyte

$<7$ days

$>0.8 * 109 / \mathrm{L}$

Myoglobin

D-Dimer

Total Points

Rate for developing severe COVID-19 pneumonia

\begin{tabular}{llllllllll}
\hline$<0 \mathrm{ng} / \mathrm{ml}$ & & & & & \\
\hline \\
\hline
\end{tabular}

Figure I Nomogram of the mNCP-SPI. A score of 165 was considered the cutoff.

+68.00466 (if time from symptom onset to admission $\geq 7$ days)

+58.67925 (if lymphocytes $\leq 0.8 \times 10^{9} / \mathrm{L}$ )

+92.53336 (if myoglobin $\geq 90 \mathrm{mg} / \mathrm{L}$ )

+100 (if $\mathrm{D}$-dimer $\geq 0.5 \mathrm{mg} / \mathrm{L}$ )

The rate of development of severe NCP according to the nomogram is shown in Figure 1.

For the total study population (348 patients), this model showed a $c$-statistic of 0.747 (95\% CI 0.682 0.814 , Figure 2), and sensitivity and specificity were 0.765 and 0.644 , respectively, under a score cutoff of 165. For the 2,000 bootstrap samples, the AUROC of the training set was 0.753 (95\% CI $0.680-0.826)$, while that of the testing set was 0.738 (95\% CI $0.688-0.788)$. Validation was also performed using fivefold crossvalidation in the derivation cohort, which revealed accuracy of 0.7573 (95\% CI $0.7095-0.8163, p=0.021)$. The mNCP-SPI was a significantly stronger predictor of severity than CURB-65 ( $\mathrm{n}=348$, AUROC 0.747 vs 0.538; $p<0.001$; Figure 2 ). NRI of the $\mathrm{mNCP}$-SPI was also improved over CURB-65 (NRI 0.421, 95\% CI 0.$365-0.488 ; p=0.028)$.

\section{Discussion}

A novel predictive score was established based on 348 patients with mNCP in our study, among which 68 (19.5\%) developed severe NCP during hospitalization. This score included five variables: time from symptom onset to admission $\geq 7$ days, lymphocyte count $\leq 0.8 \times 10^{9} / \mathrm{L}$, D-dimer

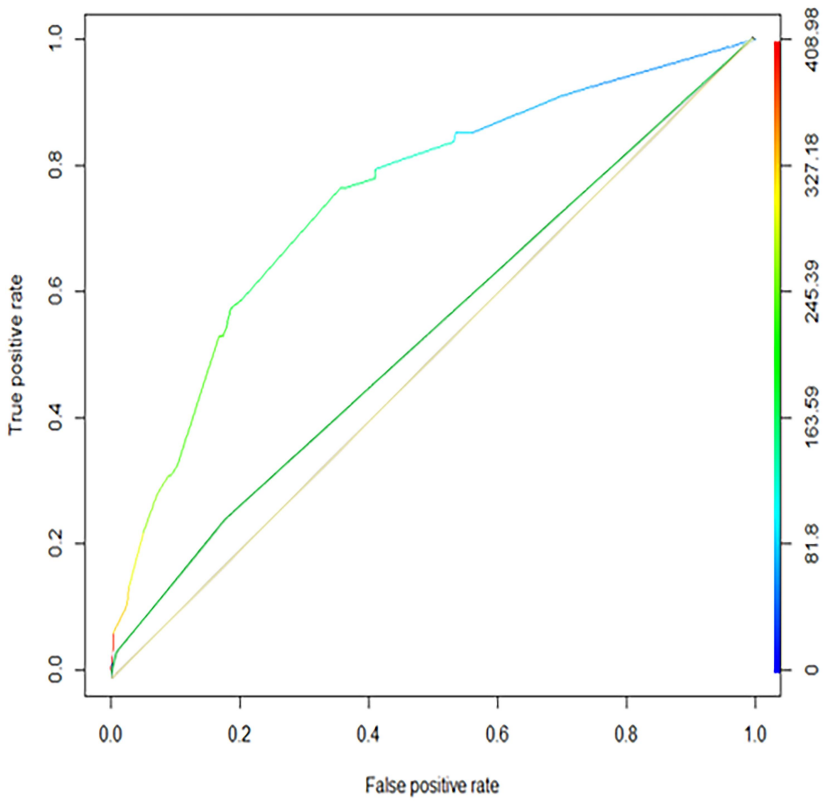

Figure 2 ROC curve and comparison between mNCP-SPI and CURB-65 scores on admission. This model showed c-statistics for the $\mathrm{mNCP}$-SPI of 0.747 , with sensitivity and specificity 0.764 and 0.644 , respectively, under the cutoff of I65. Different levels of the $\mathrm{mNCP}-\mathrm{SPI}$ are shown by color: blue the lowest and red the highest. The ROC curve of CURB-65 is presented in green, and the reference line of 0.5 is yellow.

$\geq 0.5 \mathrm{ug} / \mathrm{L}$, myoglobin $\geq 90 \mathrm{ng} / \mathrm{mL}$, and history of diabetes. This model showed sensitivity of 0.765 , specificity of 0.644 , and AUROC of 0.747 .

During hospitalization, most patients had marked lymphopenia, and nonsurvivors developed more severe lymphopenia over time. ${ }^{14}$ From the 99 patients initially reported, of the nine who died, eight had lymphopenia. ${ }^{1}$ 
Data from 1,099 patients showed that severe cases had more prominent laboratory abnormalities (ie, leukopenia, lymphopenia, thrombocytopenia) than nonsevere NCP cases. ${ }^{15}$ Consistently with these studies, our model also revealed that lymphocyte count $\leq 0.8 \times 10^{9} / \mathrm{L}$ was a risk factor for developing severe pneumonia. Previous studies have shown that invasion of viral particles in severe SARS-CoV infection can damage the cytoplasmic components of lymphocytes and that lymphocyte apoptosis may lead to lymphopenia in severe Middle East Respiratory Syndrome-CoV infection. This phenomenon is also reflected in our research. Counts and absolute values of T-lymphocyte subsets (such as $\mathrm{CD}^{+}, \mathrm{CD}^{+}$, and $\mathrm{CD}^{+}$) in the severe group were lower than those in the nonsevere group.

Aggravation of pneumonia may trigger a cytokine storm, presenting an increase in both proinflammatory and anti-inflammatory cytokines, thereby causing depletion of immune cells and decrease in lymphocytes. It has been reported that serum IL6 levels are significantly higher in patients with COVID-19 pneumonia, ${ }^{16}$ and IL6 after tocilizumab may distinguish nonsurvivors from survivors and further support the choice of deeper targeting of IL6. ${ }^{17}$ Although there was no difference in IL6 between the mild and severe groups in our study, the indices in both groups were significantly higher than normal (normally $<3.4 \mathrm{pg}$ / $\mathrm{mL}$ with Immulite 2000 reagent), suggesting extensive presence of cytokine storms in NCP patients.

Time from the onset of symptoms to admission $\geq 7$ days was an independent risk factor for developing severe NCP. Data of 1,099 patients in 31 provinces/cities also showed that time from symptom onset to developing pneumonia was significantly different between the severe group and the nonsevere group and the duration of symptoms in the severe group longer. ${ }^{15}$ A clinical study of 138 hospitalized patients with NCP showed that time from onset to dyspnea was 5 days, 7 days to hospital admission, and 8 days to acute respiratory distress syndrome. ${ }^{18}$ This could be due to the inflammatory storm reaching a peak in 5-7 days in patients with delayed treatment, and which lead to multiple-organ damage. Therefore, early identification and timely treatment are important.

Among the multiple-organ damage caused by inflammatory-factor storms and depletion of $\mathrm{T}$ lymphocytes, myocardial damage is often a factor that is easy to ignore in patients without previous heart disease. The causes of myocardial damage by NCP are the virus infection leading directly to cardiomyocyte lysis or damage to myocardial blood vessels, immuno damage to $\mathrm{T}$ cells leading to viral myocarditis, acidosis or respiratory alkalosis causing myocardial damage, hypoxemia leading to myocardial ischemia and necrosis. The combination of myoglobin and other myocardial enzyme indices, such as troponin I and creatine kinase MB can sensitively and specifically reflect myocardial damage at an early stage over a wide diagnostic time, ${ }^{19}$ and is also an important parameter to evaluate the prognosis of pulmonary infection.

The storm of inflammatory factors can also cause disorders in coagulation. D-dimer is a specific molecular marker produced by the hydrolysis of plasmin after crosslinking fibrin monomer with activating factor XII. When the D-dimer concentration in plasma increases, it indicates that the body has active thrombosis and secondary hyperfibrinolysis, which is common in various thrombotic diseases, such as deep-vein thrombosis, pulmonary embolism, and disseminated intravascular coagulation. ${ }^{20}$ Studies have also pointed out that hypercoagulability is a common manifestation of severe pneumonia. ${ }^{21}$ Plasma D-dimer levels are closely related to inflammatory levels ${ }^{22}$ and severity of pneumonia, and are valuable for prognostic prediction. ${ }^{23}$ It has also been observed that SARS patients with disseminated intravascular coagulationhad severer disease and higher risk of death. ${ }^{24} \mathrm{~A}$ recent study showed that prothrombin time and D-dimer level on admission were higher in ICU patients than non-ICU patients. ${ }^{7}$ The laboratory findings of 1,099 patients with COVID-19 acute respiratory distress syndrome also showed that compared with the nonsevere group, the proportion of $\mathrm{D}$-dimer $\geq 0.5 \mathrm{mg} / \mathrm{L}$ in the severe group is higher. ${ }^{15} \mathrm{D}$-dimer $\geq 0.5 \mathrm{mg} / \mathrm{L}$ is also a risk factor for developing severe NCP in this model, as damage to the vascular endothelium caused by cytokine storms can lead to the up-regulation of coagulation factors and downregulation of physiological anticoagulation pathways. ${ }^{25}$

Diabetes is a common chronic disease, and immunofunction in diabetic patients is at high risk of various acute and chronic infections. ${ }^{26} \mathrm{~A}$ history of diabetes and ambient hyperglycemia are independent predictors of death and morbidity in SARS patients, and metabolic control may improve the prognosis of SARS patients. ${ }^{27}$ A history of diabetes is also an independent risk factor for severe NCP in our model. Studies have reported that in patients with diabetes, anti-inflammatory macrophages can be transformed into proinflammatory macrophages, and regulatory T cells can be transformed into $\mathrm{T}_{\mathrm{H}} 1$ and $\mathrm{T}_{\mathrm{H}} 17 \mathrm{CD} 4^{+}$helper T lymphocytes. $^{28,29}$ This may be an explanation for why diabetic patients tend to become severer. What is more, 
infection can also cause sharp fluctuations in blood glucose in diabetic patients, which will adversely affect prognosis. $^{28}$ Therefore, great attention should be paid to the management of blood glucose in diabetic patients with NCP.

Clinical prediction rules for pneumonia, such as CURB65 , have been widely used to evaluate community-acquired pneumonia, but lack specific indicators of viral infection and are not sensitive enough to predict the severity and prognosis of viral pneumonia. In our study, it has been verified that our model has better predictive ability than CURB-65, and all variables included are easy to acquire on admission. Meanwhile, previous clinical studies have shown that risk factors, such as chronic underlying diseases, combined bacterial infections, and high-dose glucocorticoid use, are all associated with poor prognosis of viral infections. ${ }^{18,30} \mathrm{We}$ have also established a six-parameter MuLBSTA score predicting mortality of virus infection, ${ }^{31}$ but it is unclear whether the model is suitable for novel $\mathrm{CoV}$, considering the rapid progression and low rate of bacterial coinfection of patients with COVID-19. A prior study has also reported on the CALL scoring system with four clinical parameters proposed by Ji et al, yet the sample size was small and needs further validation. ${ }^{32}$ Our study provides another prediction tool with considerable ability. At the same time, our results shed light on the importance of early intervention, glycemic control, and preventive anticoagulant therapy in this long-lasting epidemic.

Our research has limitations. First of all, due to the multicenter source of patients, some data were missing during the collection of medical records, such as early anti-infection treatment, the specific usage of glucocorticoids, and control of patients' blood glucose. At the same time, our study did not include external validation, due to the limited number of mNCP patients and lack of other independent data, which is rather important and should be further confirmed. In summary, we found that the mNCPSPI score had significant predictive ability for development of severe pneumonia among patients with mNCP. This could assist physicians who are currently treating NCP on the front line and may help to identify potential candidates for ICU and further medical support.

\section{Ethics Approval}

This study was approved by the Coordinating Ethics Committee of Ruijin Hospital, affiliated with Shanghai Jiao Tong University School of Medicine (2017-205), and was conducted in accordance with the Declaration of Helsinki.

\section{Author Contributions}

All authors made a significant contribution to the work reported, whether in the conception, study design, execution, acquisition of data, analysis, and interpretation, or in all these areas; took part in drafting, revising, or critically reviewing the article; gave final approval to the version to be published; have agreed on the journal to which the article has been submitted; and agree to be accountable for all aspects of the work.

\section{Funding}

The funders had no role in the design or conduct of the study; collection, management, analysis, or interpretation of the data; preparation, review, or approval of the manuscript; or decision to submit the manuscript for publication. This work was supported by the National Key R\&D Program of China (2017YFC1309700 and 2017YFC1309701), Shanghai Shenkang Hospital Development Center Clinical Science and Technology Innovation Project (SHDC12018102), Shanghai Key Discipline for Respiratory Diseases (2017ZZ02014), and the Clinical NCP Focused Research Program (Translational Medicine Funding of Shanghai Jiao Tong University). This work was also funded in part by a grant from the Innovative Research Team from of high-level local universitiesin Shanghai and by the Institute of Respiratory Disease, School of Medicine, Shanghai Jiao Tong University.

\section{Disclosure}

The authors declare that they have no competing interests in this work.

\section{References}

1. Chen N, Zhou M, Dong X, et al. Epidemiological and clinical characteristics of 99 cases of 2019 novel coronavirus pneumonia in Wuhan, China: a descriptive study. Lancet. 2020;395(10223):50 7-513. doi:10.1016/S0140-6736(20)30211-7

2. Lu H, Stratton CW, Tang YW. Outbreak of pneumonia of unknown etiology in Wuhan, China: the mystery and the miracle. J Med Virol. 2020;92(4):401-402. doi:10.1002/jmv.25678

3. Lu R, Zhao X, Li J, et al. Genomic characterisation and epidemiology of 2019 novel coronavirus: implications for virus origins and receptor binding. Lancet. 2020;395(10224):565-574. doi:10.1016/S0140-6736 (20)30251-8

4. Zhou P, Yang XL, Wang XG, et al. A pneumonia outbreak associated with a new coronavirus of probable bat origin. Nature. 2020. doi: 10.1038/s41586-020-2012-7

5. Zhu N, Zhang D, Wang W, et al. A novel coronavirus from patients with pneumonia in China, 2019. N Engl J Med. 2020;382(8):727-733. doi:10.1056/NEJMoa2001017 
6. Wu A, Peng Y, Huang B, et al. Genome composition and divergence of the novel coronavirus (2019-nCoV) originating in China. Cell Host Microbe. 2020

7. Huang C, Wang Y, Li X, et al. Clinical features of patients infected with 2019 novel coronavirus in Wuhan, China. Lancet. 2020;395 (10223):497-506. doi:10.1016/S0140-6736(20)30183-5

8. Jin YH, Cai L, Cheng ZS, et al. A rapid advice guideline for the diagnosis and treatment of 2019 novel coronavirus (2019-nCoV) infected pneumonia (standard version). Military Med Res. 2020;7 (1):4. doi:10.1186/s40779-020-0233-6

9. Pan Y, Guan H, Zhou S, et al. Initial CT findings and temporal changes in patients with the novel coronavirus pneumonia (2019-nCoV): a study of 63 patients in Wuhan, China. Eur Radiol. 2020;30:3306-3309. doi:10.1007/s00330-020-06731-x

10. Ahnert P, Creutz P, Horn K, et al. Sequential organ failure assessment score is an excellent operationalization of disease severity of adult patients with hospitalized community acquired pneumonia - results from the prospective observational PROGRESS study. Crit Care. 2019;23(1):110. doi:10.1186/ s13054-019-2316-x

11. Chen JH, Chang SS, Liu JJ, et al. Comparison of clinical characteristics and performance of pneumonia severity score and CURB-65 among younger adults, elderly and very old subjects. Thorax. 2010;65(11):971-977. doi:10.1136/thx.2009.129627

12. Martos-Benitez FD, Cordero-Escobar I, Soto-Garcia A, et al. APACHE II score for critically ill patients with a solid tumor: A reclassification study. Rev Esp Anestesiol Reanim. 2018;65 (8):447-455

13. De Grooth HJ, Geenen IL, Girbes AR, et al. SOFA and mortality endpoints in randomized controlled trials: a systematic review and meta-regression analysis. Crit Care. 2017;21(1):38. doi:10.1186/ s13054-017-1609-1

14. Guan W-J, Ni Z-Y, Hu Y, et al. Clinical characteristics of 2019 novel coronavirus infection in China. MedRxiv. 2020. doi:10.1101/ 2020.02.06.20020974

15. Hendgen-Cotta UB, Flogel U, Kelm M, et al. Unmasking the Janus face of myoglobin in health and disease. J Exp Biol. 2010;213(Pt 16):2734-2740. doi:10.1242/jeb.041178

16. Chen G, Wu D, Guo W, et al. Clinical and immunological features of severe and moderate coronavirus disease 2019. J Clin Invest. 2020:137244. doi:10.1172/JCI137244.

17. Quartuccio L, Sonaglia A, Pecori D, et al. Higher levels of IL-6 early after tocilizumab distinguish survivors from non-survivors in COVID-19 pneumonia: a possible indication for deeper targeting IL-6. J Med Virol. 2020. doi:10.1002/jmv.26149

18. Wang D, Hu B, Hu C, et al. Clinical characteristics of 138 hospitalized patients with 2019 novel coronavirus-infected pneumonia in Wuhan, China. JAMA. 2020;323:1061. doi:10.1001/jama.2020.1585

19. Stein PD, Hull RD, Patel KC, et al. D-dimer for the exclusion of acute venous thrombosis and pulmonary embolism: a systematic review. Ann Intern Med. 2004;140(8):589-602. doi:10.7326/00034819-140-8-200404200-00005

Infection and Drug Resistance

\section{Publish your work in this journal}

Infection and Drug Resistance is an international, peer-reviewed openaccess journal that focuses on the optimal treatment of infection (bacterial, fungal and viral) and the development and institution of preventive strategies to minimize the development and spread of resistance. The journal is specifically concerned with the epidemiology of
20. Milbrandt EB, Reade MC, Lee M, et al. Prevalence and significance of coagulation abnormalities in community-acquired pneumonia. $\mathrm{Mol}$ Med. 2009;15(11-12):438-445. doi:10.2119/molmed.2009.00091

21. Querol-Ribelles JM, Tenias JM, Grau E, et al. Plasma d-dimer levels correlate with outcomes in patients with community-acquired pneumonia. Chest. 2004;126(4):1087-1092. doi:10.1378/chest.126.4.1087

22. Quartuccio L, Sonaglia A, McGonagle D, et al. Profiling COVID-19 pneumonia progressing into the cytokine storm syndrome: results from a single Italian Centre study on tocilizumab versus standard of care. J Clin Virol. 2020;129:104444. doi:10.1016/j.jcv.2020.104444

23. Wong RS, Wu A, To KF, et al. Haematological manifestations in patients with severe acute respiratory syndrome: retrospective analysis. BMJ (Clinical Research Ed). 2003;326(7403):13 58-1362.

24. Idell S. Coagulation, fibrinolysis, and fibrin deposition in acute lung injury. Crit Care Med. 2003;31(4 Suppl):S213-20. doi:10.1097/01. CCM.0000057846.21303.AB

25. Hodgson K, Morris J, Bridson T, et al. Immunological mechanisms contributing to the double burden of diabetes and intracellular bacterial infections. Immunology. 2015;144(2):171-185. doi:10.1111/imm. 12394

26. Yang JK, Feng Y, Yuan MY, et al. Plasma glucose levels and diabetes are independent predictors for mortality and morbidity in patients with SARS. Diabet Med. 2006;23(6):623-628. doi:10.1111/j.14645491.2006.01861.x

27. Meshkani R, Vakili S. Tissue resident macrophages: key players in the pathogenesis of type 2 diabetes and its complications. Clin Chim Acta. 2016;462:77-89.

28. Xia C, Rao X, Zhong J. Role of T lymphocytes in type 2 diabetes and diabetes-associated inflammation. $J$ Diabetes Res. 2017;20 17:6494795. doi:10.1155/2017/6494795

29. Hine JL, De Lusignan S, Burleigh D, et al. Association between glycaemic control and common infections in people with type 2 diabetes: a cohort study. Diabet Med. 2017;34(4):551-557. doi:10. 1111/dme.13205

30. Viasus D, Pano-Pardo JR, Pachon J, et al. Factors associated with severe disease in hospitalized adults with pandemic (H1N1) 2009 in Spain. Clin Microbiol Infect. 2011;17(5):738-746. doi:10.1111/ j.1469-0691.2010.03362.x

31. Guo L, Wei D, Zhang X, et al. Clinical features predicting mortality risk in patients with viral pneumonia: the MuLBSTA score. Front Microbiol. 2019;10:2752.

32. Ji D, Zhang D, Xu J, et al. Prediction for progression risk in patients with COVID-19 pneumonia: the CALL score. Clin Infect Dis. 2020: pii: ciaa414. doi:10.1093/cid/ciaa414. antibiotic resistance and the mechanisms of resistance development and diffusion in both hospitals and the community. The manuscript management system is completely online and includes a very quick and fair peerreview system, which is all easy to use. Visit http://www.dovepress.com/ testimonials.php to read real quotes from published authors. 